\title{
Cyclic variation of Doppler power as a function of hematocrit and vessel wall compliance
}

\section{Misaridis, Athanasios; Shung, K.K.}

Published in:

I E E E International Ultrasonics Symposium. Proceedings

Link to article, DOI:

10.1109/ULTSYM.1998.765235

Publication date:

1998

Document Version

Publisher's PDF, also known as Version of record

Link back to DTU Orbit

Citation $(A P A)$ :

Misaridis, A., \& Shung, K. K. (1998). Cyclic variation of Doppler power as a function of hematocrit and vessel wall compliance. I E E E International Ultrasonics Symposium. Proceedings, 2, 1533-1536.

https://doi.org/10.1109/ULTSYM.1998.765235

\section{General rights}

Copyright and moral rights for the publications made accessible in the public portal are retained by the authors and/or other copyright owners and it is a condition of accessing publications that users recognise and abide by the legal requirements associated with these rights.

- Users may download and print one copy of any publication from the public portal for the purpose of private study or research.

- You may not further distribute the material or use it for any profit-making activity or commercial gain

- You may freely distribute the URL identifying the publication in the public portal 


\title{
CyClic VARIATION OF DOPPler POWER AS A FunCtion OF HEMATOCRIT AND VESSEl WALl COMPLIANCE
}

\author{
Thanassis X. Misaridis \\ Center for Fast Ultrasound Imaging, Department of Information Technology, Build. 344, \\ Technical University of Denmark, DK-2800 Lyngby, Denmark. \\ K. Kirk Shung \\ The Bioengineering Program, The Pennsylvania State University, University Park, PA 16802, USA.
}

\begin{abstract}
In order to better understand the relationship between the power of the Doppler spectrum and blood flow abnormalities, a systematic study of all factors that may affect the Doppler power is required. In this study, three important factors were investigated in vitro using a mock flow loop: a) the pulsation rate of the flow, b) the hematocrit of blood and c) the vessel wall compliance. The results may be useful for the development of a noninvasive method for assessing blood vessel wall compliance and fetal hematocrit in utero. The results show that the peak Doppler power decreases 1.5 to 2 $\mathrm{dB}$ as the pulsation rate increases from 20 to 55 beats $/ \mathrm{min}$. The cyclic variation of the Doppler power is between 2.3 and $6.7 \mathrm{~dB}$ at 20 beats $/ \mathrm{min}$, depending on the hematocrit, but it drops to 1.6-2.4 dB at 55 beats/min. Moreover, both the time delays of the cyclic variation from the peak systole and the amplitude of the peak of the power are observed to be a function of the hematocrit. This is more evident in compliant vessels at the low beat rate of 20 beats $/ \mathrm{min}$. When the vessel becomes more compliant, the Doppler power peak is shifted from the early to the late systole. These results suggested that simultaneous measurements of the time shift of the Doppler power peak relative to the velocity waveform and the magnitude of Doppler power cyclic variation may yield indication as to the value of the hematocrit and vessel compliance.
\end{abstract}

\section{INTRODUCTION}

Despite the wide use of Ultrasound routinely as a diagnostic tool, the exact dependence of the backscattered signal power on several parameters is still not completely understood. Of clinical importance is the dependence of the Doppler power on hematocrit, vessel wall compliance, and flow conditions since those factors are associated with disease states. Thus, the objective of this work is to validate our notion about the cyclic variation of the power of the Doppler spectrum and its dependencies. Another impetus for this research is to clarify and try to explain why previous in vitro experiments in the literature report different cyclic variation schemes from what has been measured in vivo. The hypothesis is whether this discrepancy can be attributed to different experimental flow conditions and possibly to the vessel wall elasticity and associated different states of red cell aggregation.

\section{Previous Results}

Previous work in this field has revealed the dependence of the Doppler power on hematocrit for red cell suspensions [1], but not for whole blood. There is also no evaluation of the effect of the elasticity of the vessel. In compliant vessels, cyclic variations in the Doppler power from whole blood have been reported [2], but no results have been presented on the effect of hematocrit. De Kroon et al. [3] acquired B-mode images with a high frequency intravascular transducer from the iliac artery and found that the echogenicity of blood followed the inverse of the flow waveform. Cloutier et al. [4] reported a similar decrease at early systole in vitro using red cell suspensions but only at a high mean velocity $(64 \mathrm{~cm} / \mathrm{s})$. At $11 \mathrm{~cm} / \mathrm{s}$ they found no significant power variation over the cycle. Further studies on whole blood [2] reported an opposite relationship at low beat rates and no changes at physiologic beat rates.

\section{Influencing Mechanisms}

Scattering from blood is a complex phenomenon affected by several parameters. Red cell aggregation appears to play a major role, especially at low shear rates [5]. The pulsatility of the flow causes continuous formation and break up of the rouleaux that will create variations on the Doppler power. Since the Doppler signal is stochastic, the scattering strength depends on the correlation among scatterers. The latter might also vary during the flow cycle. The accuracy of the spectral estinate is affected by the nonstationarity of the signal during acceleration and the associated spectral broadening.

\section{MATERIALS AND METHODS}

\section{Flow Loop}

The experimental arrangement is illustrated in Fig. 1. A piston pump (Harvard Apparatus) was used to produce the desired pulsatile flow waveform. Blood coming out from the pump flowed first to an entrance reservoir and then to the conduit. Porcine blood was used in this study because of its resemblance to human blood. Continuous mixing of 
blood in the reservoir was performed by a magnetic stirrer (Fisher Thermix Stirrer 220T) to prevent blood settling. A square-wave electromagnetic flowmeter (Carolina Medical Electronics, Model 501) was used to measure the mean flow rate. The flowmeter was calibrated for every blood sample with different hematocrits by collecting and measuring the volume of blood that passed through the flow probe over a known period of time. A micro-tip catheter pressure transducer (Millar Instruments, Inc., Model MPC-500) was mounted downstream of the acoustic window, allowing continuous monitoring of the absolute pressure of the flow loop.

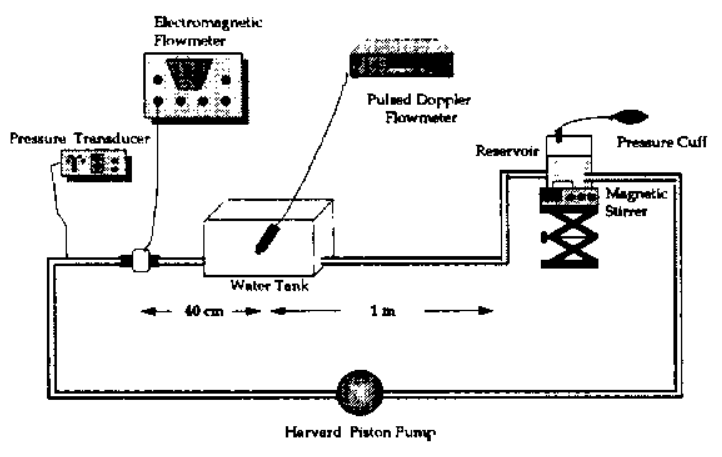

Figure I: Experimental setup for the Doppler measurements on porcine blood under pulsatile flow conditions.

A rigid polystyrene tube (Nalgene) and a flexible latex tube (Fisher Scientific) were used to mimick the blood vessels. Both tubes had a wall thickness of $1.59 \mathrm{~mm}$, inner diameter of $6.35 \mathrm{~mm}$, and a length of $118 \mathrm{~cm}$. The flow conduit was immersed horizontally in a tank filled with distilled water for acoustic coupling. In the compliant tube experiments, two rigid tubes with much larger diameter were connected externally on either sides of the tank and the elastic tube was placed inside them. The water level in the tank covered the two rigid tube outlets and therefore, the elastic tube was suspended in water and could expand freely all along its length. The calculated Womersley number $\alpha$, was found to be 3.92 at 55 beats $/ \mathrm{min}, 3.22$ at 37 beats $/ \mathrm{min}$ and 2.36 at 20 beats $/ \mathrm{min}$ for the fundamental frequency at a hematocrit of $40 \%$. Values of $\alpha$ close to 1 assure that the flow waveform does not lag the pressure gradient and the velocity remains nearly parabolic [6]. The Reynolds number gives a rough indication of the stability of laminar flow under pulsatile flow. In our loop the critical value of 2,000 corresponds to a mean velocity of $1.2 \mathrm{~m} / \mathrm{s}$. It should also be noted that the maximum velocity at the center of the vessel, associated with this mean velocity, strongly depends on the velocity profile. By adjusting the volume per stroke ejected by the pump, mean blood velocity was kept below this value.

\section{Acoustic apparatus}

The angle between the Doppler transducer and the tube was kept constant at $65^{\circ}$ for all measurements. The sample volume was positioned at the center of the vessel. The Doppler measurements were performed with a pulsed Doppler system built for laboratory purposes operating at $10 \mathrm{MHz}$ [7]. The $10 \mathrm{MHz}$ single element transducer was excited with 4 cycles of the $10 \mathrm{MHz}$ signal, at a pulse repetition frequency (PRF) of $39 \mathrm{KHz}$. The wall filter had a cut-off frequency of $200 \mathrm{~Hz}$. The power density spectrum of the audio signal in the absence of flow exhibited a decrease with frequency. The compensation was performed numerically by fitting a 10 th order polynomial to the normalized frequency response. Each Doppler frequency component was multiplied by the inverse of the normalized frequency response.

\section{Data Acquisition and Processing}

Three channels were used for acquisition of the mean flow signal, the pressure signal, and the forward output of the Doppler device. The signal from the electromagnetic flowmeter was used as the digital signal for synchronizing the acquisition from the other two channels. When the peak of the flow was detected, the Doppler audio signal was acquired at a sampling rate of $51.2 \mathrm{KHz}$ at a softwareadjustable delay after the peak of the flow. The digitized time signal was divided into 1024 -point $20 \mathrm{~ms}$ segments, a duration which is a compromise between the stationarity of the signal and a sufficient observation time to get a good spectral estimate. The mean value of the averaged time signal from each 20 -ms segment was calculated and subtracted from the signal. To decrease the spectral leakage caused by the sidelobes introduced by the finite observation time, a 1024-point Kaiser window with $\beta$ equal to 5 was applied to the data. Then, a 1024-point Fast Fourier Transform was applied on each windowed segment to calculate the power density spectrum. The procedure was repeated for 100 flow cycles and the signal originating from the same time segment relatively to the peak of the flow, was averaged.

\section{EXPERIMENTAI. RESULTS}

A series of experiments was performed using both rigid and elastic tubes under similar experimental conditions, and the dependence of the Doppler power on hematocrit was quantified. Three different pulsation rates were used: 20,37 and 55 beats $/ \mathrm{min}$. The results reported represent the mean value averaged over 4 experiments with different blood samples. The standard deviations are indicated by the error bars.

Results obtained at pulsation rates of 20 beats $/ \mathrm{min}$ from the rigid and elastic tube experiments are shown in Fig. 2. For the rigid tube, the results show a significant cyclic variation of the mean power, that increases with hematocrit. The variation increases from $2.3 \mathrm{~dB}$ at a hematocrit of $9 \%$ up to $6.8 \mathrm{~dB}$ and almost $9 \mathrm{~dB}$ at hematocrits of 25 and $36 \%$ 
respectively. The peak of the mean power slightly leads the variation of the flow but does not change with hematocrit. For the elastic tube experiments at the same beat rate, the peak of the power does not occur at the same time instant within the flow cycle as in the rigid tube case, and it is affected by the hematocrit. The results from the elastic tube experiments are summarized in Table $I$. When the beat rate was increased to 37 beats $/ \mathrm{min}$, a smaller variation of about $3.2 \mathrm{~dB}$ was found (Fig. 3). At a hematocrit of $36 \%$ the power maximum dropped more than $3 \mathrm{~dB}$ in comparison to the maximum at 20 beats $/ \mathrm{min}$. The averaged power has also dropped about $1.6 \mathrm{~dB}$. The variation becomes even smaller (less than $2.5 \mathrm{~dB}$ ) at 55 beats/min for both rigid and elastic tubes and the dependence of hematocrit becomes less clear. These results are shown in Fig. 4.
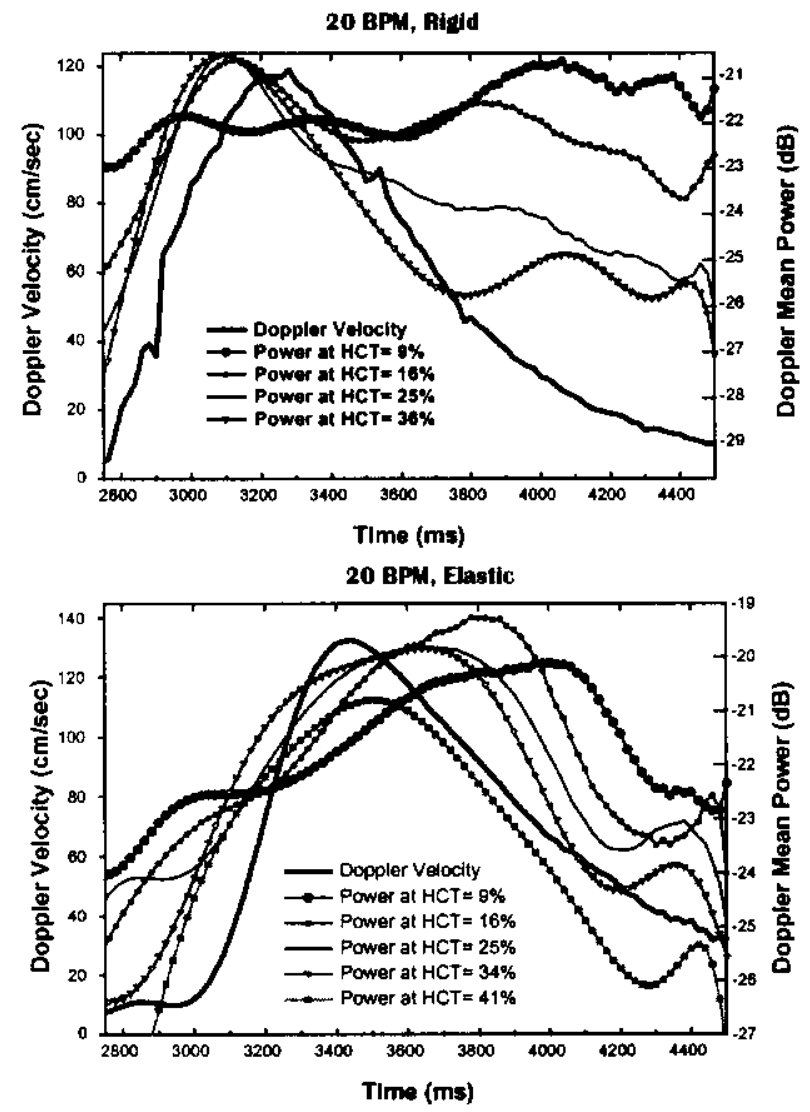

Figure 2: Mean velocity and mean Doppler power at 20 beats/min as a function of hematocrit.

\begin{tabular}{|c|c|c|c|}
\hline $\begin{array}{c}\text { HCT } \\
(\%)\end{array}$ & $\begin{array}{c}\text { Cyclic Variation } \\
(\mathrm{dB})\end{array}$ & $\begin{array}{c}\text { Peak Power } \\
(\mathrm{dB})\end{array}$ & $\begin{array}{c}\text { Delay from Peak } \\
\text { Systole (ms) }\end{array}$ \\
\hline 9 & 2.95 & -20.1 & 560 \\
16 & 4.76 & -19.3 & 300 \\
25 & 5.14 & -19.8 & 240 \\
34 & 5.73 & -19.8 & 220 \\
41 & 6.73 & -20.8 & 100 \\
\hline
\end{tabular}

Table 1: Summary of the cyclic variation of the mean Doppler power from the early to the late systole as a function of hematocrit in the elastic tube experiments at 20 beats/min.

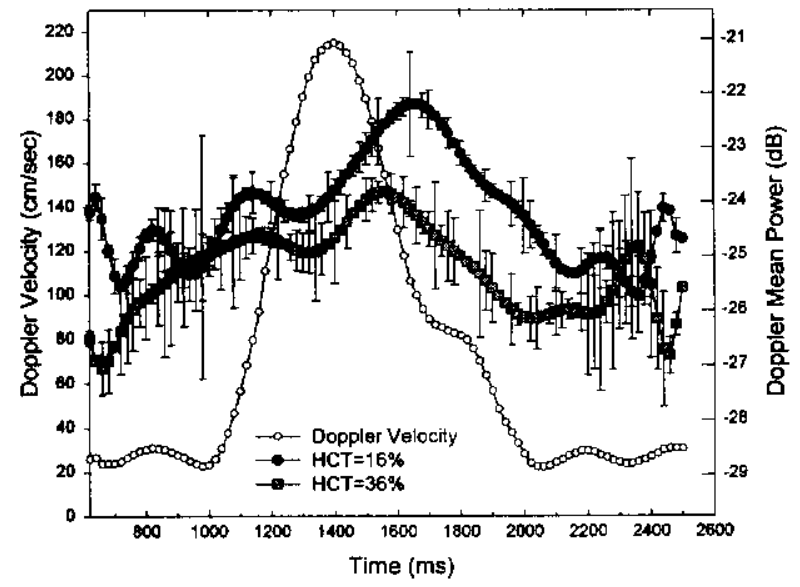

Figure 3: Mean velocity and mean Doppler power at 37 beats/min for two different hematocrits in rigid tube.
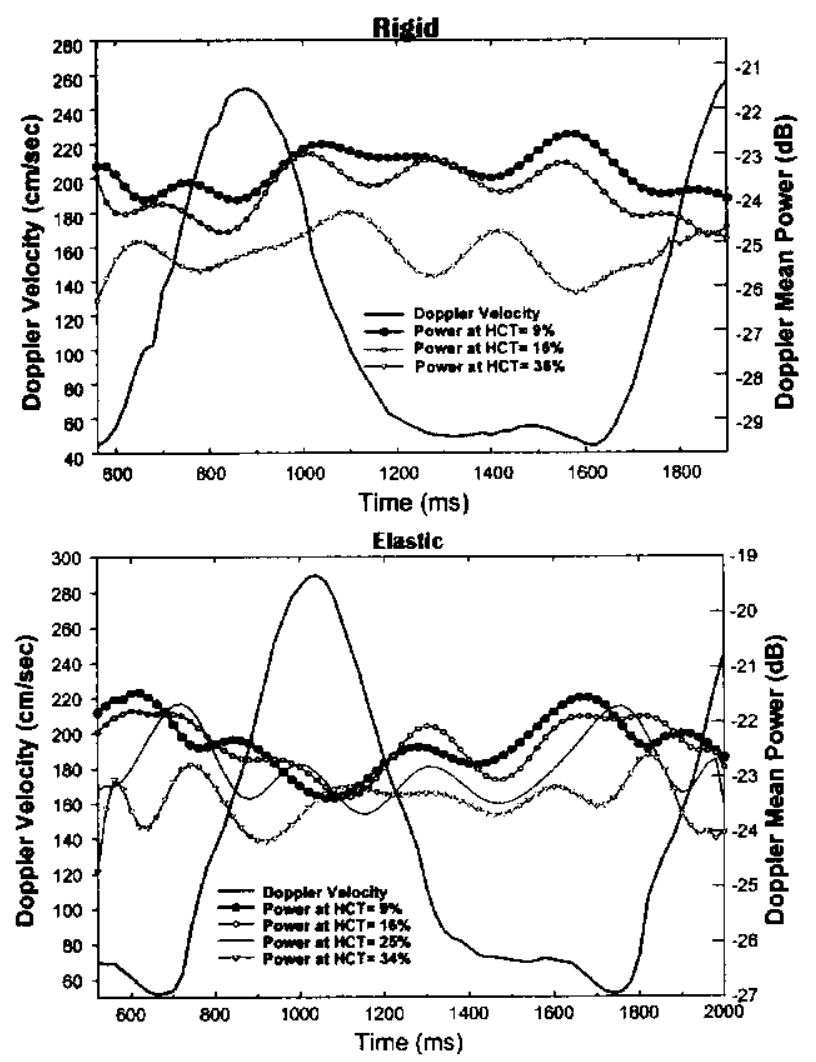

Figure 4: Mean velocity and mean Doppler power at 55 beats/min as a function of hematocrit.

\section{Discussion}

The results presented in the previous section indicate that the Doppler scattering power and its variation during the 
flow cycle strongly depend on the pulsation rate. The precise reasons for the observed behavior may not be fully understood, but it is possible to speculate on the mechanisms that are responsible for these variations.

The long diastolic period allows the cells to be brought into contact. Scattering signals arising from adjacent cells, that do not form aggregates, interfere rather destructively, causing the overall scattering power to drop. This hypothesis explains the low power levels at the left and right ends of the graphs. This argument is supported by the fact that the power drops with increased hematocrit, since the destructive interference gets higher when the particle concentration increases. During acceleration, the aligned cells probably form rouleax, that causes the power to rise until the shear stresses become high enough to break them again after deceleration.

The tube compliance may affect the shear rate within the vessel at a given instant of the flow cycle and therefore the level of aggregation. The elastic vessel expands and contracts in response to the pulsatile flow. Just before acceleration, the vessel shrinks and the cells are pulled away from the center prohibiting aggregation. This might be the reason why the increased power during acceleration that occurred in rigid tubes was not seen. At late systole, the vessel diameter is stabilized again giving rise to power peaks. After the vessel contraction higher hematocrit values favor aggregation and the effect of elasticity cannot stop the formation of aggregates. As a result the peak of the power is moving to the right, close to the peak systole as hematocrit increases. The drop in the power during late systole is again more evident for higher hematocrits due to higher destructive interference as was postulated before.

When the beat rate increases, the aggregation tendency decreases, and the averaged power over the flow cycle drops. The cyclic variation becomes smaller, since the formation and destruction of the cell aggregates are reduced.

In the rigid tube experiments, the tubes used to connect the rigid main conduit with the pump were not perfectly rigid. The non-uniform elasticity of the loop and the reflections due to the impedance mismatch have introduced a finite wave velocity. Wave reflection occurs even in uniform tubes, due to the terminal vascular impedance, similarly to that seen in the transmission line theory [6]. A rough estimation of the phase velocity was made by measuring the time delay between the flow peak detected by the electromagnetic flowmeter and the Doppler device. However, the phase velocity varies along the length of the tube [6] and this measurement only gives a rough indication of the hemodynamic conditions that are present. In the set of experiments performed at 20 beats/min, the time delay did not change significantly from the rigid to the elastic tube experiments $(1.6-1.9 \mathrm{~m} / \mathrm{s})$. When a different flow loop was constructed entirely from elastic tubes, a drop in the Doppler power was measured at 37 beats/min, associated with the pressure drop during the vessel contraction, similar to that measured in vivo [3]. The phase velocity decreased approximately by a factor of 2 in this loop. Thus, it is very likely that the cyclic variation of the Doppler power is also associated with the propagation velocity along the vessel's length. These preliminary results are not presented here.

\section{CONCLUSIONS}

In this study, the presence of a cyclic variation in the echogenicity has been demonstrated in vitro in rigid and elastic vessels only under certain flow conditions. The different variation schemes observed at different pulsation rates are likely to be associated with the aggregation states of whole blood during the cycle as well as with changes in the correlation among cells caused by differences in flow dynamics. At physiological high-rate pulsatile flow, RBC aggregation seems to play a lesser role.

Commercial scanners display either the velocity estimate or the Doppler power. The elastic tube experiments at low beat rates showed a correlation between hematocrit and the position of the mean Doppler power maximum relative to the peak of the flow. Therefore, simultaneous measurement of velocity and mean Doppler power may give an indication of the value of hematocrit, by measuring the delay of the power maximum from peak systole.

The importance of hemodynamic parameters on the cyclic variation of the Doppler power has been identified. Examination of parameters such as the amplitude and phase angle of the vascular impedance and the phase velocity at different sites may yield more evidence as to the origin of the cyclic variation in Doppler power.

\section{ACKNOWLEDGMEN'TS}

This work has been supported by NIH grant \# HL28452.

\section{REFERENCES}

[1] G. Cloutier, K. K. Shung, "Cyclic Variation of the Power of Ultrasonic Doppler Signals Backscattered by Polystyrene Microspheres and Porcine Erythrocyte Suspensions", IEEE Transnctions on Biomedical Engineering, Vol. 40, No. 9, pp. 953962 (1993).

[2] S. J. Wu and K. K. Shung. "Cyclic Variation of Doppler Power from Whole Blood under Pulsatile Flow", Ulirasound in Med. \& Biol., Vol. 22, No 7, pp. 883-894 (1996).

[3] G. M. de Kroon, C. J. Slager, W. J. Gussenhoven, P. W. Serruys, J. R. T. C. Roelandt and N. Bom, "Cyclic Changes of Blood Echogenicity in High-Frequency Ultrasound", Ultrasound in Med. \& Bial. Vol. 17, No. 7, pp. 723-728 (1991).

[4] G. Cloutier, K. K. Shung, "Study of Red Cell Aggregation in Pulsatile Flow from Ultrasonic Doppler Power Measurements", Biorheology30, pp. 443-461 (1993).

[5] K. K. Shung, G. Cloutier, C. C. Lim, "The Effects of Hematocrit, Shear Rate and Turbulence on Ultrasonic Doppler Spectrum from Blood", IEEE Trans. Biomedl. Eng., vol. 39, No. 5, p. 462 (1992).

[6] W. R. Milnor, "Hemodynamics", 2nd Edition, Williams \& Wilkins (1989).

[7] C. J. Hartley, Reference Manual for the $10 \mathrm{MHz}$ Pulsed Doppler Flowmeter, Baylor College of Medicine, Houston, TX (1980). 